\title{
$C M$-Selectors for Pairs of Oppositely Semicontinuous Multifunctions and Some Applications to Strongly Nonlinear Inclusions
}

\author{
Hôǹg Thái Nguyêñ, M. Juniewicz and J. Ziemińska
}

\begin{abstract}
We present a new approximate joint selection theorem which unifies Michael's theorem (1956) on continuous selections and Cellina's theorem (1969) on continuous $\varepsilon$-approximate selections. More precisely, we show that, given a convex-valued $H$-upper semicontinuous multifunction $F$ and a convex-closed-valued lower semicontinuous multifunction $G$ with $F(x) \cap$ $G(x) \neq \emptyset$, one can find a continuous function $f$ which is both a selection of $G$ and an $\varepsilon$ approximate selection of $F$. We also prove a parametric version of this theorem for multifunctions $F$ and $G$ of two variables $(s, u) \in \Omega \times X$ where $\Omega$ is a measure space. Using this selection theorem, we obtain an existence result for elliptic systems involving a vector Laplacian and a strongly nonlinear multi-valued right-hand side, subject to Dirichlet boundary conditions.

Keywords: Joint, continuous and $\varepsilon$-approximate selectors, $H$-upper and lower semicontinuous multifunctions, multifunctions satisfying one-side estimates, Dirichlet elliptic inclusions, multi-valued elliptic systems, problems with strong non-linearities, with lack of compactness and with critical exponents
\end{abstract}

AMS subject classification: Primary 54 C 65, 54 C 60, 28 B 20, 35 R 70, secondary 47 H 04, 34 A 60

\section{Introduction}

The first purpose of this paper is to present a new continuous joint selection theorem (Theorem 2.1) which unifies two known theorems due to E. A. Michael [13] in 1956 and to A. Cellina [7] in 1969. More precisely, we prove that if $F$ is an $H$-upper semicontinuous convex-valued multifunction from $X$ to $2^{Y}, G$ is a lower semicontinuous convex-closedvalued multifunction from $X$ to $2^{Y}$, and $F(x) \cap G(x) \neq \emptyset$ for all $x \in X$, then there exists a $C M$-selector for the pair $(F, G)$, i.e. there exists a continuous function which is both a selector for $G$ (as in Michael's theorem) and an $\varepsilon$-approximate selector for $F$ (as in Cellina's theorem). In the case $G(x) \equiv Y$ Theorem 2.1 reduces to the Cellina

All authors: Szczecin University, Res. Center of H-Hura-Sciences \& Inst. Math., ul. Wielkopolska 15, 70-451 Szczecin, Poland

e-mail: rchhurasci@his-hu.ac.vn, nguyenht@sus.univ.szczecin.pl nhthaimt@uoo.univ.szczecin.pl (November-June), nguyenht@his-hu.ac.vn (July-October) juniewic@sus.univ.szczecin.pl and jolka@sus.univ.szczecin.pl 
theorem for $F$. In the case $F(x) \equiv Y$ it reduces to the Michael theorem for $G$. In the general case our theorem can be interpreted as an "intermediate" theorem between the Michael and Cellina theorems.

The notion of $C M$-selectors and the problem of their existence find motivation in our research on the existence of solutions of strongly nonlinear multi-valued problems: nonlinear Hammerstein multi-valued equations (inclusions) and elliptic boundary value problems with strongly nonlinear multi-valued right-hand sides $F$ satisfying some onesided estimates (the sign condition, the generalized sign condition, the Hammerstein one-sided estimate, etc.). We observed that in such a case each one-sided estimate generates in the multi-valued setting some pair $(F, G)$, where the multifunction $G$ is lower semicontinuous (see Theorems 2.2 and 3.1). The strong nonlinearity of $F(s, x)$ means that we have to consider problems involving $F$ in the cases of the lack of compactness and of critical exponents in the exact non-compact Sobolev embedding theorems (Sobolev's and Pokhozaev-Trudinger's).

The second part of the present paper (Sections 4 and 5) is therefore devoted to some applications of $\varepsilon$-approximate $C M$-selectors as well as to the study of the simplest multi-valued strongly nonlinear problem (inclusion). To this end, we prove a simple parametric version of Theorem 2.1 (see Theorem 3.1) for multifunctions $F$ and $G$ of two variables $(s, x) \in \Omega \times X$ where $\Omega$ is a measure space. Next we apply the result to constructing a sequence of single-valued strongly nonlinear Dirichlet problems $-\Delta u(s)=$ $f_{n}(s, u(s))$ approximating the original multi-valued strongly nonlinear Dirichlet problem $-\Delta u(s) \in F(s, u(s))$ in an "appropriate" sense such that the functions $f_{n}(s, x)$ satisfy the same one-sided estimate as the multifunction $F(s, x)$ (see our construction of $C M$ relaxations $f_{n}(s, x)$ in (5.1) - (5.2) of Step 1 in Section 5; cf. with usual truncated relaxations $f_{n}(s, x)$ in the proof of [4: Theorem 2] and [15: Formula (28)]).

Finally we formulate and prove an existence theorem (Theorem 4.1) for the above multi-valued strongly nonlinear problem (the simplest inclusion with lack of compactness), emphasizing seven main steps characteristic of our weak convergence analysis via the use of the above $C M$-relaxations (see Steps 1 - 7 in Section 5).

By the way, it is interesting to notice that in the proof of a recent result of $\mathrm{Hu}$ and Papageorgiou [10] on a generalization of Browder's degree for strongly nonlinear elliptic inclusions of $(S)_{+}$type there is a gap in their construction of approximate single-valued scalar functions $g_{\varepsilon}(\cdot, \cdot)$ (see [10: p. 244 ${ }^{18}$, where in fact it is impossible to use "line segments to make continuous connections" for defining their auxiliary function $\eta_{\delta}^{*}(r)$ ). This gap can be closed by using our "applied" $\varepsilon$-approximate $C M$-selection Theorems 2.2 and 3.1 together with Remark $2.1 /(2)$ of Section 2.

\section{2. $C M$-Selectors}

For the convenience of the reader, we give the basic definitions and notations following [2, $5]$. Let $(X, \rho)$ be a metric space. For $x \in X, M \subset X$ and $\varepsilon>0$ we denote by $d(x, M)=$ $\inf \{\rho(x, y): y \in M\}$ the distance from $x$ to $M$, by $U_{\varepsilon}(M)=\{y \in X: d(y, M)<\varepsilon\}$ the $\varepsilon$-neighbourhood of $M$ and by $B(x, r)=B_{X}(x, r)$ the open ball with center $x$ and radius $r$. The distance in the product $X \times Y$ of metric spaces is defined by $d\left((x, y),\left(x_{1}, y_{1}\right)\right)=$ 
$\max \left\{\rho_{X}\left(x, x_{1}\right), \rho_{Y}\left(y, y_{1}\right)\right\}$. We assume that each multifunction considered has nonempty values, unless stated to the contrary. The graph of a multifunction $F: X \rightarrow 2^{Y}$ is the set $\operatorname{Gr} F=\{(x, y) \in X \times Y: y \in F(x)\}$. If $A \subset X$, then $F(A)$ denotes the set $\cup_{x \in A} F(x)$.

Let $X, Y$ be metric spaces. A multifunction $F: X \rightarrow 2^{Y}$ is called

- upper or lower semicontinuous at $x_{0}$ if for any open set $V \subset Y$ with $F\left(x_{0}\right) \subset V$ or $F\left(x_{0}\right) \cap V \neq \emptyset$ one can find an open neighbourhood $U \subset X$ of $x_{0}$ such that $F(x) \subset V$ or $F(x) \cap V \neq \emptyset$, respectively, for all $x \in U$.

- upper or lower semicontinuous, if it is upper or lower semicontinuous, respectively, at every $x \in X$;

- H-upper or H-lower semicontinuous at $x_{0}$ if for any $\varepsilon>0$ one can find $\delta>0$ such that $F\left(B\left(x_{0}, \delta\right)\right) \subset U_{\varepsilon}\left(F\left(x_{0}\right)\right)$ or $F\left(x_{0}\right) \subset U_{\varepsilon}(F(x))$, respectively, for all $x \in B\left(x_{0}, \delta\right)$.

- H-upper or $H$-lower semicontinuous, if it is $H$-upper or $H$-lower semicontinuous at every $x \in X$, respectively;

If $F$ is upper semicontinuous, then it is $H$-upper semicontinuous; the converse is true if $F$ takes compact values. If $F$ is $H$-lower semicontinuous, then $F$ is lower semicontinuous; the converse is true if $F$ takes compact values.

If $Y$ is a normed space, we denote by conv $D$ and $\overline{\text { conv }} D$ the convex hull and the closed convex hull of a subset $D$ of $Y$, respectively.

The main purpose of this section is to prove a theorem, which is intermediate between two famous continuous selection theorems: the Michael theorem [13] and the Cellina theorem [7]. To formulate it, there is a need for a new notion which we immediately introduce.

Definition 2.1. Let $F, G: X \rightarrow 2^{Y}$ be two multifunctions, where $X$ and $Y$ are metric spaces, and let $\varepsilon>0$ be an arbitrary positive number. By an $\varepsilon$-approximate $C M$-selector for the pair $(F, G)$ we mean a continuous function $f: X \rightarrow Y$ which is both a selector for $G$ (i.e., $f(x) \in G(x)$ for all $x \in X$ ) and an $\varepsilon$-approximate selector ( $\varepsilon$-selector in short) for $F$ (i.e. $\operatorname{Gr} f \subset U_{\varepsilon}(\mathrm{Gr} F)$ ).

Remark. If $Y$ is a normed space, then $f: X \rightarrow Y$ is an $\varepsilon$-selector for $F$ if and only if $f(x) \in F\left(B_{X}(x, \varepsilon)\right)+B_{Y}(0, \varepsilon)$ for all $x \in X$.

Theorem 2.1. Let $X$ be a metric space and $Y$ a Banach space. Assume that $F, G: X \rightarrow 2^{Y}$ are multifunctions, $F H$-upper semicontinuous with convex values and $G$ lower semicontinuous with closed convex values, and such that $F(x) \cap G(x) \neq \emptyset$ for all $x \in X$. Then for every $\varepsilon>0$ there exists an $\varepsilon$-approximate $C M$-selector for the pair $(F, G)$.

Proof. The proof of Theorem 2.1 will be carried out in two steps.

Step 1: Suppose first that $Y$ is a normed space and $G: X \rightarrow 2^{Y}$ has convex values only. We claim that then for every $\varepsilon_{1}, \varepsilon_{2}>0$ there exists a continuous map $f: X \rightarrow Y$ such that Gr $f \subset U_{\varepsilon_{1}}(\operatorname{Gr} F), f(x) \in U_{\varepsilon_{2}}(G(x))$ for every $x \in X$, and $f(X) \subset \operatorname{conv} F(X)$.

For the proof fix $\varepsilon_{1}, \varepsilon_{2}>0$. Let $y_{x}$ be an arbitrary element of $F(x) \cap G(x)$ with $x \in X . \quad F$ is $H$-upper semicontinuous, so for $x \in X$ there is $\delta_{1}(x)>0$ such that 
$\delta_{1}(x)<\varepsilon_{1}$ and $F\left(B\left(x, \delta_{1}(x)\right)\right) \subset U_{\varepsilon_{1}}(F(x))$. The multifunction $G$ is lower semicontinuous, therefore for $x \in X$ there exists $\delta_{2}(x)>0$ such that $B\left(y_{x}, \varepsilon_{2}\right) \cap G\left(x^{\prime}\right) \neq \emptyset$, i.e. $y_{x} \in U_{\varepsilon_{2}}\left(G\left(x^{\prime}\right)\right)$ for $x^{\prime} \in B\left(x, \delta_{2}(x)\right)$. Denote $\delta(x)=\min \left\{\delta_{1}(x), \delta_{2}(x)\right\}$ and $U_{x}=B\left(x, \frac{1}{2} \delta(x)\right)$ for $x \in X$. Since $\left(U_{x}\right)_{x \in X}$ is an open covering of the metric space $X$ and $X$ is paracompact by the Stone theorem [12], we can find a locally finite refinement $\left(W_{i}\right)_{i \in I}$ of $\left(U_{x}\right)_{x \in X}$ and a continuous partition of unity $\left(\phi_{i}\right)_{i \in I}$ subordinate to $\left(W_{i}\right)_{i \in I}$. For each $i \in I$ choose $x_{i} \in X$ such that $\phi_{i} \equiv 0$ on $X \backslash U_{x_{i}}$. Denote $\delta\left(x_{i}\right)=\delta_{i}, U_{x_{i}}=U_{i}$ and $y_{x_{i}}=y_{i}$ for $i \in I$. Define the function $f: X \rightarrow Y$ by $f(x)=\sum_{i \in I} \phi_{i}(x) y_{i}$. Evidently, $f$ is continuous, and as $f(x)$ is a convex combination of elements of $F(X)$, we have $f(x) \in \operatorname{conv} F(X)$ for every $x \in X$.

Observe that $f$ is an $\varepsilon_{1}$-selector of $F$. Indeed, let $x \in X$ and denote $I(x)=\{i \in I$ : $\left.\phi_{i}(x) \neq 0\right\}$. The set $I(x)$ is finite and we have $f(x)=\sum_{i \in I(x)} \phi_{i}(x) y_{i}$. Define $j \in I(x)$ so that $\delta_{j}=\max _{i \in I(x)} \delta_{i}$. If $i \in I(x)$, then $\phi_{i}(x)>0$ and hence $x \in U_{i}$. Now

$$
\rho\left(x_{i}, x_{j}\right) \leq \rho\left(x_{i}, x\right)+\rho\left(x, x_{j}\right)<2\left(\frac{1}{2} \delta_{j}\right)=\delta_{j},
$$

so $x_{i} \in B\left(x_{j}, \delta_{j}\right)$, and therefore $y_{i} \in U_{\varepsilon_{1}}\left(F\left(x_{j}\right)\right)$ for $i \in I(x)$. Consequently, $f(x) \in$ $U_{\varepsilon_{1}}\left(F\left(x_{j}\right)\right)$ as the $\varepsilon$-neighbourhood $U_{\varepsilon_{1}}\left(F\left(x_{j}\right)\right)$ of the convex set $F\left(x_{j}\right)$ in the normed space $Y$ is convex. On the other hand, $x \in B\left(x_{j}, \varepsilon_{1}\right)$ because $\delta_{j}<\varepsilon_{1}$. Finally, $(x, f(x)) \in U_{\varepsilon_{1}}(\operatorname{Gr} F(x))$ for every $x \in X$, i.e. $f$ is an $\varepsilon_{1}$-selector for $F$.

For the proof of the remaining part of our statement, let again $x \in X$. If $i \in I(x)$, then $x \in U_{i}$ and hence $y_{i} \in U_{\varepsilon_{2}}(G(x))$. Therefore $f(x)=\sum_{i \in I(x)} \phi_{i}(x) y_{i} \in U_{\varepsilon_{2}}(G(x))$ as the set $G(x)$ and hence also $U_{\varepsilon_{2}}(G(x))$ is convex.

Step 2: Assume now that $Y$ is even a Banach space and that $G$ takes closed convex values. We claim that for every $\varepsilon>0$ there exists a $C M$-selector $f$ for the pair $(F, G)$. Indeed, fix $\varepsilon>0$. By Step 1 there exists a continuous map $f_{1}: X \rightarrow Y$ such that $\operatorname{Gr} f_{1} \subset U_{\frac{\varepsilon}{2}}(\operatorname{Gr} F)$ and $f_{1}(x) \in U_{\frac{\varepsilon}{2}}(G(x))$ for $x \in X$. Consider the multifunction $G_{1}: X \rightarrow 2^{Y}$ defined by $G_{1}(x)=\overline{G(x) \cap B\left(f_{1}(x), \frac{\varepsilon}{2}\right)}$. Of course, $G_{1}$ has non-empty closed convex values. Moreover, $G_{1}$ is lower semicontinuous (see, e.g., [5: Proposition 1.1.5]). Thus, by the famous Michael theorem, $G_{1}$ has a continuous selector $f: X \rightarrow Y$. Note that $f$ is also a selector for $G$ as $G_{1}(x) \subset \overline{G(x)}=G(x)$ for $x \in X$.

It remains to show that $\operatorname{Gr} f \subset U_{\varepsilon}(\operatorname{Gr} F)$. Indeed, let $x \in X$. Since $\left(x, f_{1}(x)\right) \in$ $U_{\frac{\varepsilon}{2}}(\operatorname{Gr} F)$, we have $\rho\left(x^{\prime}, x\right)<\frac{\varepsilon}{2}$ and $\rho\left(y, f_{1}(x)\right)<\frac{\varepsilon}{2}$ for some $x^{\prime} \in X$ and $y \in F\left(x^{\prime}\right)$. Hence $\rho(y, f(x)) \leq \rho\left(y, f_{1}(x)\right)+\rho\left(f_{1}(x), f(x)\right)<\frac{\varepsilon}{2}+\frac{\varepsilon}{2}=\varepsilon$, because $f(x) \in G_{1}(x) \subset$ $\bar{B}\left(f_{1}(x), \frac{\varepsilon}{2}\right)$. Thus we have $d\left((x, f(x)),\left(x^{\prime}, y\right)\right)<\varepsilon$, and consequently $f$ is an $\varepsilon$-selector for $F$

Remarks 2.1. 1) Theorem 2.1 is true also in the more general setting of Cellina's Theorem 1 from [8], i.e. when $X$ is a paracompact, uniform space with countable base (in particular, metric space) and $Y$ is a complete metric, locally convex space (i.e. Fréchet space). The $L_{p}$-decomposable nonconvex-valued version of the theorem is valid too (cf. [6]; the results were announced in H. T. Nguyêñ [14] and are accepted for publication in $[16])$.

2) From Theorem 2.1 follows in addition the possibility to construct a $C M$-selector $f$ such that $f(a)=f_{0}(a) \quad(a \in A)$, where $A \subset X$ is a fixed closed set (in particular, $A$ is a fixed finite or closed countable set) and $f_{0}: A \rightarrow Y$ is a fixed continuous 
function such that $f_{0}(a) \in F(a) \cap G(a) \quad(a \in A)$. For a proof put $G_{0}(x)=\left\{f_{0}(x)\right\}$ for $x \in A$ and $G_{0}(x)=G(x)$ for $x \notin A$. By [13] (see also [5]), $G_{0}$ is lower semicontinuous just as $G$. Applying the statement of Theorem 2.1 for the pair $\left(F, G_{0}\right)$, we get its $C M$ selector $f$, which clearly is a $C M$-selector for the pair $(F, G)$ with the additional property $f(x)=f_{0}(x) \quad(x \in A)$. The existence of $\varepsilon$-approximate selectors with this property, for an $H$-upper semicontinuous multifunction (as in Cellina's selection theorem [7]) seems to be unnoticed before (see recent references in the books [5, 11], and recent papers, for example [10]), although it is well-known that a lower semicontinuous multifunction of Michael's theorem has a continuous selector satisfying the additional property.

The following "applied" $\varepsilon$-approximate $C M$-selection theorem (and it together with the above Remark 2.1/(2)) is an example of how Theorem 2.1 can be applied to constructing $\varepsilon$-approximate continuous selectors satisfying some additional conditions.

Theorem 2.2. Let $X$ be a Banach space and $X^{*}$ be its dual. Assume that $F: X \rightarrow$ $2^{X^{*}}$ is a $H$-upper semicontinuous multifunction with convex values and that $g: X \rightarrow \mathbb{R}$ is a continuous non-negative function. Define $G: X \rightarrow 2^{X^{*}}$ by

$$
G(x)= \begin{cases}\left\{y \in X^{*}:\langle x, y\rangle \leq g(x)\right\} & \text { if } x \neq 0 \\ X^{*} & \text { if } x=0 \text { and } g(0)>0 \\ \{0\} & \text { if } x=0 \text { and } g(0)=0 .\end{cases}
$$

Assume that $F(x) \cap G(x) \neq \emptyset$ for all $x \in X$. Then $G$ is lower semicontinuous, and the pair $(F, G)$ has an $\varepsilon$-approximate $C M$-selector for every $\varepsilon>0$.

Proof. It suffices to show that $G$ satisfies the assumptions of Theorem 2.1. It is clear that $G$ has non-empty closed convex values. It remains to show that it is lower semicontinuous. Indeed, assume that $G$ is not lower semicontinuous at some $x_{0} \in X$. Then there exist an open set $V \subset X^{*}$ such that $G\left(x_{0}\right) \cap V \neq \emptyset$ and a sequence $\left(x_{n}\right) \subset X \backslash\{0\}$, which converges to $x_{0}$ and such that $G\left(x_{n}\right) \cap V=\emptyset$ for $n \in \mathbb{N}$. Therefore, for every $y \in V$ we have $\left\langle x_{n}, y\right\rangle>g\left(x_{n}\right)$ for $n \in \mathbb{N}$ and hence $\left\langle x_{0}, y\right\rangle \geq g\left(x_{0}\right)$ by the continuity of $\langle\cdot, \cdot\rangle$ and $g$. This is a contradiction if $g(0)>0$ and $x_{0}=0$.

Assume now that $x_{0} \neq 0$ and take $y_{0} \in G\left(x_{0}\right) \cap V$. Then $B\left(y_{0}, r\right) \subset V$ for some $r>0$. From the above it follows that $\left\langle x_{0}, y_{0}\right\rangle=g\left(x_{0}\right)$. On the other hand, by the classical Hahn-Banach theorem [9], there exists $z \in X^{*}$ with $\|z\|=1$ and $\left\langle x_{0}, z\right\rangle=\left\|x_{0}\right\| \neq 0$. Then for $y=y_{0}-\frac{r}{2} z$ we have $y \in B\left(y_{0}, r\right) \subset V$ and $\left\langle x_{0}, y\right\rangle=g\left(x_{0}\right)-\frac{r}{2}\left\|x_{0}\right\|<g\left(x_{0}\right)$ which is a contradiction.

The lower semicontinuity of $G$ at $x_{0}=0$ when $g(0)=0$ is obvious, since in this case by definition $G(0)=\{0\}$. So if $G(0) \cap V \neq \emptyset$ where $V$ is open, then $0 \in V$. But of course $0 \in G(x)$ for every $x \in X$, hence $G(x) \cap V \neq \emptyset$ for every $x \in X$

Remark that the inequality $\langle x, y\rangle \leq g(x)$ is called in the literature one-sided estimate. 


\section{3. $C M$-selectors for multifunctions of two variables}

Let $(\Omega, \mathfrak{A}, \mu)$ be a measure space with a complete, $\sigma$-finite measure $\mu$ on a $\sigma$-algebra $\mathfrak{A}$. Let $X$ and $Y$ be separable complete metric spaces. A multifunction $F: \Omega \rightarrow$ $2^{X}$ with closed values is called measurable if the set $\{x \in X: F(x) \cap U \neq \emptyset\}$ is measurable for every open subset $U$ of $X$. For other equivalent notions of measurability of multifunctions see, e.g., $[2,5]$.

We recall that $f: \Omega \times X \rightarrow Y$ is called a Carathéodory function if $f(s, \cdot)$ is continuous for almost all $s \in \Omega$ and $f(\cdot, x)$ is measurable for all $x \in X$. Following e.g. [2], a multifunction $F: \Omega \times X \rightarrow 2^{Y}$ is called $H$-upper Carathéodory if $F(s, \cdot)$ is $H$-upper semicontinuous for almost all $s \in \Omega$ and $F(\cdot, x)$ is measurable for all $x \in X$.

Further, a multifunction $F: \Omega \times X \rightarrow 2^{Y}$ is called $(\bmod 0)$-measurable if $F(\cdot, \cdot)$ is measurable on $\left(\Omega \backslash D_{0}\right) \times X$ with respect to the algebra $\mathfrak{A} \times \mathcal{B}(X)$ where $D_{0}$ is some measurable set with $\mu\left(D_{0}\right)=0$ and $\mathcal{B}(X)$ is the algebra of all Borel subsets of $X$. More information concerning multifunctions of two variables can be found e.g. in [2].

The following "applied" $\varepsilon$-approximate $C M$-selection theorem is a parametric version of Theorem 2.2. Note that Remark 2.1/(2) is valid also for Theorem 3.1, and is useful in applications.

Theorem 3.1. Let $F: \Omega \times \mathbb{R}^{m} \rightarrow 2^{R^{m}}$ be an H-upper Carathéodory and $(\bmod 0)$ measurable multifunction, taking convex compact values. Further, let $g: \Omega \times \mathbb{R}^{m} \rightarrow \mathbb{R}$ be a non-negative Carathéodory function, define $G: \Omega \times \mathbb{R}^{m} \rightarrow 2^{\mathbb{R}^{m}}$ by

$$
G(s, x)= \begin{cases}\left\{y \in \mathbb{R}^{m}:\langle x, y\rangle \leq g(s, x)\right\} & \text { if } x \neq 0 \\ \mathbb{R}^{m} & \text { if } x=0 \text { and } g(s, 0)>0 \\ \{0\} & \text { if } x=0 \text { and } g(s, 0)=0\end{cases}
$$

for any $s \in \Omega$, and assume that $F(s, x) \cap G(s, x) \neq \emptyset$ for almost all $s \in \Omega$ and all $x \in \mathbb{R}^{m}$. Then for every positive measurable function $\varepsilon: \Omega \rightarrow \mathbb{R}_{+}$there exists a Carathéodory function $f: \Omega \times \mathbb{R}^{m} \rightarrow \mathbb{R}^{m}$ such that $f(s, \cdot)$ is a $C M$-selector for the pair $(F(s, \cdot), G(s, \cdot))$ with respect to $\varepsilon(s)>0$ for almost all $s \in \Omega$.

Proof. Let $\varepsilon: \Omega \rightarrow \mathbb{R}_{+}$be an arbitrary measurable positive function. Define $\hat{F}(s, x)=F\left(s, \bar{B}\left(x, \frac{1}{2} \varepsilon(s)\right)+\bar{B}\left(0, \frac{1}{2} \varepsilon(s)\right)\right.$ for $s \in \Omega$ and $x \in \mathbb{R}^{m}$. It is easy to show that $\hat{F}(s, \cdot)$ is $H$-upper semicontinuous and has closed values for almost all $s \in \Omega$. We contend also that $\hat{F}(\cdot, x)$ is measurable for all $x \in X$. In fact, it is known that the properties of $F$ ensure that the multifunction $s \mapsto F(s, Z(s))$ is measurable for every measurable multifunction $Z: \Omega \rightarrow 2^{\mathbb{R}^{m}}$ (see, e.g., [2]). Taking $Z(s) \equiv \bar{B}\left(x, \frac{1}{2} \varepsilon(s)\right)$ for $s \in \Omega$ and fixed $x \in \mathbb{R}^{m}$ we obtain that the multifunction $F\left(\cdot, \bar{B}\left(x, \frac{1}{2} \varepsilon(\cdot)\right)\right)$, and hence also $\hat{F}(\cdot, x)$ is measurable.

Denote $H(s, x)=\hat{F}(s, x) \cap G(s, x) \quad\left(s \in \Omega, x \in \mathbb{R}^{m}\right)$ and let $C\left(\mathbb{R}^{m}, \mathbb{R}^{m}\right)$ be the separable metric space of all continuous functions from $\mathbb{R}^{m}$ into $\mathbb{R}^{m}$, with the topology of uniform convergence on compact sets. From Theorem 2.2 it follows that $H(s, \cdot)$ has a continuous selector for almost all $s \in \Omega$, so the multifunction $\Phi: \Omega \rightarrow 2^{C\left(\mathbb{R}^{m}, \mathbb{R}^{m}\right)}$ defined by $\Phi(s)=\left\{f \in C\left(\mathbb{R}^{m}, \mathbb{R}^{m}\right): f(x) \in H(s, x)\right.$ for all $\left.x \in \mathbb{R}^{m}\right\}$ has a.e. nonempty values. We noted earlier that $\hat{F}(s, \cdot)$ is $H$-upper semicontinuous for almost all 
$s \in \Omega$, and therefore for almost all $s \in \Omega$ and for any continuous function $f: \mathbb{R}^{m} \rightarrow \mathbb{R}^{m}$ the implication

$$
f(r) \in \hat{F}(s, r)\left(\forall r \in \mathbb{Q}^{m}\right) \quad \Longrightarrow \quad f(x) \in \hat{F}(s, x)\left(\forall x \in \mathbb{R}^{m}\right)
$$

is valid. In view of this fact, and because $H$ takes closed values, we can write

$$
\begin{aligned}
\operatorname{Gr} \Phi & =\left\{(s, f) \in \Omega \times C\left(\mathbb{R}^{m}, \mathbb{R}^{m}\right): d(f(x), H(s, x))=0\left(\forall x \in \mathbb{R}^{m}\right)\right\} \\
& =\left\{(s, f) \in \Omega \times C\left(\mathbb{R}^{m}, \mathbb{R}^{m}\right): d(f(r), H(s, r))=0\left(\forall r \in \mathbb{Q}^{m}\right)\right\} \\
& =\bigcap_{r \in \mathbb{Q}^{m}}\left\{(s, f) \in \Omega \times C\left(\mathbb{R}^{m}, \mathbb{R}^{m}\right): d(f(r), H(s, r))=0\right\} .
\end{aligned}
$$

Clearly, $H(\cdot, x)$ is measurable for all $x \in \mathbb{R}^{m}$, so for each $r$ of the countable set $\mathbb{Q}^{m}$ the function $(s, f) \mapsto d(f(r), H(s, r))$ is a Carathéodory function from $\Omega \times C\left(\mathbb{R}^{m}, \mathbb{R}^{m}\right)$ into $\mathbb{R}$. Hence as is well-known (see, e.g., $[2,5])$ we have

$$
\left\{(s, f) \in \Omega \times C\left(\mathbb{R}^{m}, \mathbb{R}^{m}\right): d(f(r), H(s, r))=0\right\} \in \mathfrak{A} \otimes \mathcal{B}\left(C\left(\mathbb{R}^{m}, \mathbb{R}^{m}\right)\right)
$$

where $\mathcal{B}(M)$ is the algebra of all Borel subsets of a metric space $M$. It follows that $\operatorname{Gr} \Phi \in \mathfrak{A} \otimes \mathcal{B}\left(C\left(\mathbb{R}^{m}, \mathbb{R}^{m}\right)\right)$. By the von Neumann-Aumann selection theorem (see [5]) $\Phi$ has a measurable selector $h: \Omega \rightarrow C\left(\mathbb{R}^{m}, \mathbb{R}^{m}\right)$. Set $f(s, x)=(h(s))(x)$. Then $f$ is a Carathéodory function with the desired properties

Theorem 3.1 (and a more general parametric version of Theorem 2.1) can also be proved in the framework of Fréchet or Banach spaces (by a different but rather complicated technique). Its various modifications cover multi-valued versions of many generalized Hammerstein one-sided estimates and all generalized sign conditions.

\section{The Dirichlet problem for multi-valued elliptic differential systems with strong non-linearities}

Let $\Omega$ be a bounded domain in $\mathbb{R}^{n}(n \geq 2)$ and $F: \Omega \times \mathbb{R}^{m} \rightarrow 2^{\mathbb{R}^{m}}$ some multifunction of two variables $(s, u) \in \Omega \times \mathbb{R}^{m}$. We shall consider the problem

$$
\left.\begin{array}{rl}
-\Delta_{m} u(s) & \in F(s, u(s)) \quad \text { for a.a. } s \in \Omega \\
\left.u\right|_{\partial \Omega} & =0
\end{array}\right\}
$$

where $\Delta_{m}=(\Delta, \ldots, \Delta)$ is an $m$-vector Laplacian.

In what follows we shall denote the scalar product and norm in the Euclidean space $\mathbb{R}^{m}$ by $(\cdot, \cdot)$ and $|\cdot|$, respectively, and the scalar product and norm in the Lebesgue space $L_{2}=L_{2}\left(\Omega, \mathbb{R}^{m}\right)$ by $\langle\cdot, \cdot\rangle$ and $\|\cdot\|$, respectively. As usual, $H^{1}=H^{1}\left(\Omega, \mathbb{R}^{m}\right)$ is the Sobolev space defined by the norm $\|u\|_{1}=\|u\|+\left\|\nabla_{m} u\right\|$, while $H_{0}^{1}=H_{0}^{1}\left(\Omega, \mathbb{R}^{m}\right)$ is the closure of $C_{0}^{\infty}\left(\Omega, \mathbb{R}^{m}\right)$ with respect to this norm. Denote by $H^{-1}$ the dual space to $H_{0}^{1}$ with respect to the $L_{2}$-pairing $\langle\cdot, \cdot\rangle$. Given a Young function $M: \Omega \times \mathbb{R} \rightarrow[0,+\infty)$, 
the term Orlicz space (see, e.g., [17]) will refer to the space $L_{M}=L_{M}\left(\Omega, \mathbb{R}^{m}\right.$ ) (of all equivalence classes) of all measurable functions $u$ on $\Omega$ taking values in $\mathbb{R}^{m}$, which is equipped with the Luxemburg norm $\|u\|_{M}=\inf \left\{k>0: \int_{\Omega} M(s,\|u(s)\| / k) d s \leq 1\right\}$. In particular, we shall be interested in Orlicz spaces $X$ with the property that $X \subset L_{2} \subset X^{\prime}$ where $X^{\prime}$ denotes the Köthe associate space of $X$ (see, e.g., [4]). Remember that if $M(s, \alpha)=|\alpha|^{p} \quad(1 \leq p<+\infty)$, we get $L_{M}=L_{p}$.

Throughout this section, we denote by $Z$ the special Lebesgue or Orlicz space

$$
Z= \begin{cases}L_{\frac{2 n}{n-2}} & \text { if } n>2 \\ L_{N} & \text { if } n=2\end{cases}
$$

where $N(s, \alpha)=\exp \left(|\alpha|^{2}\right)-1$. By the Sobolev exact embedding theorem (the case $n>2$ ) and the Pokhozaev-Trudinger exact embedding theorem (the case $n=2$ ), the Sobolev space $H_{0}^{1}$ is always continuously non-compactly embedded into $Z$ (see, e.g., [18]). By e.g. [4: Lemma 1], $H_{0}^{1}$ is compactly embedded into $X$, if $X$ is an Orlicz space such that the space $Z$ is absolutely continuously embedded into the space $X$, i.e. the elements of the unit ball of $Z$ have uniformly absolutely continuous norms in $X$ :

$$
\lim _{\operatorname{mes}(D) \rightarrow 0} \sup _{\|u\|_{Z} \leq 1}\left\|P_{D} u\right\|_{X}=0
$$

Here $P_{D}$ denotes the multiplication operator by the characteristic function of a measurable set $D$. Following e.g. [2], we define the multi-valued superposition operator $N_{F}$ by

$$
N_{F}(u)=\{v: v \text { is measurable and } v(s) \in F(s, u(s)) \text { a.e. }\} .
$$

We shall use one of the following acting conditions:

(AC1) $m=1$ (i.e. the case of scalar equations), $X=Z$, and the multi-valued superposition operator $N_{F}$ acts from $Z$ into $2^{Z^{\prime}}$ where $Z^{\prime}=L_{\frac{2 n}{n+2}}$ if $n>2$ and $Z^{\prime}=L_{N^{*}}$ with $N^{*}$ the dual to the Young function $N$ if $n=2$.

(AC2) $m>1$ (i.e. the case of a system of equations), and either

(a) $n>2, Z \subset X$ strictly, the multi-valued superposition operator $N_{F}$ acts from $X$ into $2^{Z^{\prime}}, Z$ is absolutely continuously embedded into $X$

or

(b) $n=2, Z \subset X$, the multi-valued superposition operator $N_{F}$ acts from $X$ into $2^{Z^{\prime}}$, and the equality $\lim _{\text {mes }}(D) \rightarrow 0 \sup _{y \in N(x),\|x\|_{X} \leq r}\left\langle y, P_{D} z\right\rangle=0$

holds for each $z \in Z$ and $r>0$.

Later on, we denote by $\mu_{\Delta}$ the first Dirichlet eigenvalue of the Laplacian $-\Delta$ on $\Omega$. The main result for this section is the following

Theorem 4.1. Let $Z$ be the space in (4.2) and $X$ be an Orlicz space such that

$$
H_{0}^{1} \subset Z \subset X \subset L_{2} \subset X^{\prime} \subset Z^{\prime} \subset H^{-1}
$$

continuously. Suppose condition (AC1) for the case $m=1$ and condition (AC2) for the case $m>1$. Suppose in addition that the following conditions are satisfied: 
1) $F(\cdot, \cdot)$ has non-empty compact convex values and is an $H$-upper Carathéodory as well as an (mod0)-measurable multifunction.

2) For almost all $s \in \Omega$ there exists $w \in F(s, u)$ such that the one-sided inequality

$$
(u, w) \leq \gamma(u, u)+\delta(s)
$$

holds where $0<\gamma<\mu_{\Delta}$ and $\delta \in L_{1}(\Omega, \mathbb{R})$ is positive.

Then problem (4.1) has at least one solution $u_{*} \in H_{0}^{1}$.

The proof of Theorem 4.1 will be given in Section 5 .

\section{Remarks 4.1.}

1) Sufficient (and necessary) conditions guaranteeing that the multi-valued superposition (Nemytskij) operator $N_{F}$ acts as desired in conditions (AC1) - (AC2) of Theorem 4.1 are completely analogous to those for the single-valued superposition operator (for the latter case see, e.g., [4]). For example, when $m=1$ and $n>2$ we may assume the polynomial growth condition

$$
\sup _{w \in F(s, u)}|w| \leq a(s)+b|u|^{\frac{n+2}{n-2}}
$$

for some $a \in L_{\frac{2 n}{n+2}}(\Omega, \mathbb{R})$ and $b \in[0,+\infty)$; when $m=1$ and $n=2$ we may assume the analogous non-polynomial exponential growth condition, using the Young function $N$ and its dual Young function $N^{*}$. It is well-known that all the exponents in (4.6) as well as in the above non-polynomial exponential growth condition are critical (and the inclusion under consideration is non-compact-type strongly nonlinear) since they all correspond to the exact continuous non-compact embeddings in the above-mentioned Sobolev/Pokhozaev-Trudinger theorems (see the discussions for the single-valued case, e.g., in $[4,15])$.

2) The compact-type nonlinear inclusions were treated, e.g., in [3] and the references cited therein.

3) Analogous existence results are valid for more complicated strongly nonlinear inclusions such as multi-valued versions of strongly nonlinear problems, which were studied, e.g., in $[4,15]$ and the references cited therein.

Example 4.1. Let $n>2$ and $D \subset \mathbb{R}$ be a fixed closed non-empty set (finite or countable, or uncountable such as a Cantor "middle thirds" set). Put $\varphi=\varphi_{1}+\varphi_{2}$, where $\varphi_{1}(u)=|u|^{\frac{n+2}{n-2}}$ for $u \leq 0$ and $\varphi_{1}(u)=0$ for $u>0$, and $\varphi_{2}(u)=0$ for $u \in D$ and $\varphi_{2}(u)=1$ for $u \notin D$ (then the set of discontinuity points of $\varphi$ coincides with $D)$. Define [2] the so-called (Krasovskij) convexification of the discontinuous $\varphi$ by $\varphi^{*}(u)=\cap_{\eta>0} \overline{\mathrm{CO}}(\varphi([u-\eta, u+\eta]))$. Given any $h \in L_{2}(\Omega)$ with $h \notin L_{p}(\Omega)$ for all $p>n$, Theorem 4.1 allows us to state the solvability result for $(4.1)$ with $F(s, u)=\varphi^{*}(u)+h(s)$, while the example cannot be treated by [3], and papers cited therein. 


\section{Proof of Theorem 4.1}

We shall divide the proof of Theorem 4.1 into 7 steps.

Step 1: By Theorem 3.1 (if $F(s, \cdot)$ is independent of $s$, it is sufficient to apply Theorem 2.2) there exists for each $\varepsilon>0$ a Carathéodory function $g_{\varepsilon}(\cdot, \cdot): \Omega \times \mathbb{R}^{m} \rightarrow \mathbb{R}^{m}$ such that

$$
\operatorname{Gr} g_{\varepsilon}(s, \cdot) \subset\left\{(u, v) \in \mathbb{R}^{m} \times \mathbb{R}^{m}: d((u, v), \operatorname{Gr} F(s, \cdot))<\varepsilon\right\} \text { a.e. }
$$

and, moreover, $g_{\varepsilon}$ satisfies the one-sided estimate

$$
\left(u, g_{\varepsilon}(s, u)\right) \leq \gamma(u, u)+\delta(s)
$$

where $\gamma$ and $\delta(\cdot)$ are the same as in (4.5) and do not depend on $\varepsilon$. Choosing $\varepsilon_{n}=\frac{1}{n}$ we define so-called $C M$-relaxations $f_{n}$ by

$$
f_{n}(s, u)= \begin{cases}g_{\frac{1}{n}}(s, u) & \text { if }\left|g_{\frac{1}{n}}(s, u)\right| \leq n \\ n \frac{g_{\frac{1}{n}}(s, u)}{\left|g_{\frac{1}{n}}(s, u)\right|} & \text { if }\left|g_{\frac{1}{n}}(s, u)\right|>n .\end{cases}
$$

By (5.2) and (5.3), $f_{n}$ are Carathéodory functions and

$$
f_{n}(s, u)=\theta_{n}(s, u) g_{\frac{1}{n}}(s, u)
$$

where $0<\theta_{n}(s, u)<1$ and

$$
\left(u, f_{n}(s, u)\right) \leq \gamma(u, u)+\delta(s)
$$

Step 2: As is well-known (see, e.g., [18]), the operator $L$ generated by the Laplacian $-\Delta_{m}$ is continuous and invertible from $H_{0}^{1}$ into $H^{-1}$, and

$$
\langle L u, u\rangle \geq \alpha\|u\|_{1}^{2} \quad\left(u \in H_{0}^{1}\right)
$$

for some $\alpha>0$ (see, e.g., [18]). Remember that the solvability of (4.1) in $H_{0}^{1}$ means the existence of $u \in H_{0}^{1}$ and $v \in N_{F}(u)$ such that $v \in H^{-1}$ and $L u=v$. Now we consider the approximate single-valued problem in $H_{0}^{1}$

$$
\left.\begin{array}{rlr}
-\Delta_{m} u(s) & =f_{n}(s, u(s)) & \text { a.e. } \\
\left.u\right|_{\partial \Omega} & =0
\end{array}\right\}
$$

where $f_{n}$ is defined in (5.4) and satisfies (5.5). Clearly, the single-valued superposition operator $F_{n}$, where $F_{n}(x)=f_{n}(\cdot, x(\cdot))$, maps the space $L_{2}$ into itself. In the presence of (5.5) via $C M$-selections, by [4: Lemma 5], the continuous compact operator $L^{-1} F_{n}$ has a fixed point $u_{n} \in L_{2}$ (i.e. $u_{n}=L^{-1} F_{n} u_{n}$ ) such that

$$
\left\|u_{n}\right\|_{L_{2}}^{2} \leq\left\|u_{n}\right\|_{1}^{2} \leq \frac{d}{c}
$$


where $d=\|\delta(\cdot)\|_{L_{1}}$ and $c=\alpha\left(\mu_{\Delta}-\gamma\right) \mu_{\Delta}^{-1}$ do not depend on $n \in \mathbb{N}$.

Step 3: In the presence of (5.8) and (5.5) via $C M$-selections, by the same argument [4], we deduce that the inequality

$$
\int_{\Omega}\left|\left(F_{n} u_{n}(s), u_{n}(s)\right)\right| d s \leq 2 \sigma
$$

holds for $u_{n}$ from Step 2, where $\sigma=d\left(1+\frac{\gamma}{c}\right)$ does not depend on $n \in \mathbb{N}$.

Step 4: We claim additionally that for $u_{n}$ from Step 2 we have the equality

$$
\lim _{\operatorname{mes}(D) \rightarrow 0} \sup _{n}\left\langle F_{n} u_{n}, P_{D} z\right\rangle=0
$$

for each $z \in Z$. In the presence of (5.9) and (5.5) via $C M$-selections, this can be verified directly as in the single-valued case [4].

Step 5: From (5.10) via the Dunford-Pettis type $\sigma\left(Z^{\prime}, Z\right)$-weak precompactness and $\sigma\left(Z^{\prime}, Z\right.$ )-weak completeness theorems in $Z^{\prime}$ (see, e.g., $[9,16]$ ) it follows that there exist some subsequence $n_{k}$ and some $v_{*} \in Z^{\prime}$ such that $\left\langle F_{n_{k}} u_{n_{k}}, z\right\rangle \rightarrow\left\langle v_{*}, z\right\rangle$ for each $z \in Z$. Further, since $L^{-1}$ acts continuously from $H^{-1}$ into $H_{0}^{1}$ and $H_{0}^{1} \subset Z \subset Z^{\prime} \subset H^{-1}$ continuously (see (4.2)), $L^{-1}$ acts continuously from $Z^{\prime}$ into $Z$. So the dual operator $\left(L^{-1}\right)^{*}$ acts continuously from $Z^{*}$ into $\left(Z^{\prime}\right)^{*}$. Remark that $Z^{\prime} \subset Z^{*}$ continuously and $\left(Z^{\prime}\right)^{*}=\left(Z^{\prime}\right)^{\prime}=Z$, since $Z$ is a perfect space and $Z^{\prime}=\left(Z^{\prime}\right)^{0}$ (see [17]) by our choice of $Z$ in (4.2). Therefore, $\left(L^{-1}\right)^{*}$ acts continuously from $Z^{\prime}$ into $Z$, and so $\left\langle L^{-1} v, z\right\rangle=$ $\left\langle v,\left(L^{-1}\right)^{*} z\right\rangle$ for all $v, z \in Z^{\prime}$. Consequently,

$$
\left\langle L^{-1}\left(F_{n_{k}} u_{n_{k}}\right), z\right\rangle=\left\langle F_{n_{k}} u_{n_{k}},\left(L^{-1}\right)^{*} z\right\rangle \rightarrow\left\langle v_{*},\left(L^{-1}\right)^{*} z\right\rangle=\left\langle L^{-1} v_{*}, z\right\rangle
$$

for each $z \in Z^{\prime}$, as $k \rightarrow+\infty$. So $L^{-1}\left(F_{n_{k}} u_{n_{k}}\right)$ converges in the weak topology $\sigma\left(Z, Z^{\prime}\right)$ to $L^{-1} v_{*}$.

Step 6: From (5.8) and the Rellich-Kondrashov theorem (see [18]) we get that $\left\{u_{n}\right\}_{n}$ is precompact in $L_{2}$ and in measure. Therefore we may choose a subsequence of $\left\{n_{k}\right\}_{k}$, which we shall for simplicity denote again by $\left\{n_{k}\right\}$, such that $u_{n_{k}}$ converges in $L_{2}$ to $u_{*}$ and $u_{n_{k}}(s) \rightarrow u_{*}(s)$ for almost all $s \in \Omega$, for some measurable function $u_{*} \in L_{2}$. From (5.8) we get that $u_{n_{k}}, u_{*} \in Z$. From our choice of $n_{k}$, we get also that (see Step 5) $\left\langle F_{n_{k}} u_{n_{k}}, z\right\rangle \rightarrow\left\langle v_{*}, z\right\rangle \quad(k \rightarrow+\infty)$ for each $z \in Z=\left(Z^{\prime}\right)^{*}$, i.e. $F_{n_{k}} u_{n_{k}} \rightarrow v_{*}$ in the weak topology $\sigma\left(Z^{\prime},\left(Z^{\prime}\right)^{*}\right)$. Invoking, e.g., [1: Theorem 8] about sequential strongweak continuous dependence, we get $v_{*} \in N_{F}\left(u_{*}\right)$. We draw attention of the reader to the fact that for to apply the theorem it is crucial that in definition (5.3) of the functions $f_{n}$ there is involved property (5.1) of $C M$-selectors. Let us remark here that in the single-valued case of $F(\cdot, \cdot)$ one can get by the Nemytskij theorem (see $[4,15,17])$ also the convergence in measure of $F_{n_{k}} u_{n_{k}}$ to $N_{F}\left(u_{*}\right)$ (and so one can get immediately $\left.v_{*}=N_{F}\left(u_{*}\right)\right)$; in the multi-valued case this is not true.

Step 7: From Step 2 we get $u_{n_{k}}=L^{-1}\left(F_{n_{k}} u_{n_{k}}\right)$. From the results of Steps 5 and 6 we then obtain $u_{*}=L^{-1} v_{*}$ (since by the well-known Hahn-Saks-Vitali theorem[9] the limit in measure and the $\sigma\left(Z, Z^{\prime}\right)$-weak limit coincide in $\left.Z\right)$ and $v_{*} \in N_{F}\left(u_{*}\right)$. Therefore $u_{*} \in L^{-1} N_{F}\left(u_{*}\right)$, and so $L u_{*} \in N_{F}\left(u_{*}\right)$ 
Acknowledgement. The paper is supported by the All-Polish Research Foundation (KBN, Warsaw), in particular, under the grant Nr 0705/P3/94/06 for the first author. The authors acknowledge the generous financial support from the Foundation for their joint research project "Nonlinear Set-Valued Analysis and Its Applications to Integral/Differential Inclusions" (since 1994). The present paper is an extended exposition with complete proofs for some our new results announced recently in H. T. Nguyêñ [14] (in September 1997).

The authors are grateful also for the constructive critical remarks of the reviewers, which influenced the shape of the revised version.

\section{References}

[1] Amrani, A., Castaing, C. and M. Valadier: Méthodes de troncature appliquées à des problèmes de convergence faible ou forte dans $L^{1}$. Arch. Rat. Mech. Anal. 117 (1992), $167-191$.

[2] Appell, J., De Pascale, E., Nguyêñ, H. T. and P. P. Zabrejko: Multivalued Superpositions (Diss. Math.: Vol. 345). Warsaw: Polish Acad. Sci. 1995, pp. 1 - 97.

[3] Appell, J., De Pascale, E., Nguyêñ, H. T. and P. P. Zabrejko: Nonlinear Integral Inclusions of Hammerstein type. Top. Methods Nonlin. Anal. 5 (1995), 109 -122.

[4] Appell, J., Nguyêñ, H. T. and P. P. Zabrejko: General existence theorems for quasilinear elliptic systems without monotonicity. J. Math. Anal. Appl. 145 (1990), 26 - 38.

[5] Aubin, J.-P. and A. Cellina: Differential Inclusions. Set Valued Maps and Viability Theory. Berlin et al.: Springer-Verlag 1984.

[6] Bressan, A. and G. Colombo: Extensions and selections of maps with decomposable values. Studia Math. 90 (1988), 69 - 86.

[7] Cellina, A.: Approximation of set-valued functions and fixed point theorems. Ann. Mat. Pura Appl. 82 (1969), $17-24$.

[8] Cellina, A. and A. Lasota: A new approach to the definition of topological degree for multi-valued mappings. Atti Accad. Naz. Lincei 47 (1969), $434-440$.

[9] Dunford, N. and J. Schwartz: Linear Operators. Vol. I: General Theory. New York: Intersci. Publ. 1958.

[10] Hu, Sch. Ch. and N. S. Papageorgiou: Generalizations of Browder's degree theory. Trans. Amer. Math. Soc. 347 (1995), 233 - 259.

[11] $\mathrm{Hu}, \mathrm{Sch}$. Ch. and N. S. Papageorgiou: Handbook of Multivalued Analysis. Vol. I: Theory. Dordrecht: Kluwer Acad. Publ. 1997.

[12] Kuratowski, K.: Topology. Vol. I and II. New York: Acad. Press 1966 and 1968.

[13] Michael, E. A.: Continuous Selections. Part I. Ann. Math. 63 (1956), 361 - 381.

[14] Nguyêñ, H. T.: Equations and inclusions with strong nonlinearities. In: Abstracts of Invited Lectures on V-th Vietnamese Math. Conf. (Hanoi, 17 - 20 September, 1997). Hanoi: Viet. Acad. Sci. 1997, p. 88.

[15] Nguyêñ, H. T.: Existence theorems for boundary value problems for strongly nonlinear elliptic systems. Z. Anal. Anw. 18 (1999), 585 - 610.

[16] Nguyen, H. T., Juniewicz, M. and J. Zieminska: CM-selectors for pairs of oppositely semicontinuous multi-valued maps with $L_{p}$-decomposable values. Studia Math. (accepted). 
[17] Rao, M. M. and Z. D. Ren: Theory of Orlicz Spaces. New York: Marcel Dekker 1991.

[18] Zeidler, E.: Nonlinear Functional Analysis and its Applications. Parts 1 - 4. Berlin et al.: Springer-Verlag 1986 - 1998.

Received 10.04.1999; in revised form 17.12.1999 